\title{
CoASH and CoASSG Levels in Lungs of Hyperoxic Rats as Potential Biomarkers of Intramitochondrial Oxidant Stresses
}

\author{
DONOUGH J. O’DONOVAN, LYNETTE K. ROGERS, DONALD K. KELLEY, STEPHEN E. WELTY, \\ PATRICIA L. RAMSAY, AND CHARLES V. SMITH \\ Department of Pediatrics, Baylor College of Medicine, One Baylor Plaza, Houston, Texas 77030, U.S.A. \\ [D.J.O., P.L.R.], Children's Research Institute, Children's Hospital, and the Department of Pediatrics, \\ The Ohio State University, Columbus, Ohio 43205, U.S.A. [L.K.R., S.E.W., C.V.S.]; and Austin Medical \\ Education Program, Austin, Texas 78701, U.S.A. [D.K.K.]
}

\begin{abstract}
Coenzyme A (CoASH) is compartmentalized preferentially in the mitochondria, and CoASH and its mixed disulfide with glutathione (CoASSG) undergo thiol/disulfide exchange reactions with glutathione (GSH) and glutathione disulfide (GSSG) in vitro. We measured CoASH and CoASSG in freeze-clamped lung tissues from Fischer-344 and Sprague-Dawley rats maintained in room air or exposed to $>95 \% \mathrm{O}_{2}$ for $48 \mathrm{~h}$ to test the hypothesis that oxidant stresses on lung thiol status would be observed in the CoASH/CoASSG redox couple, suggesting oxidant stress responses in the mitochondria. Lung tissue concentrations of CoASSG in the Fischer-344 rats declined from $0.89 \pm$ 0.15 to $0.51 \pm 0.13 \mathrm{nmol} / \mathrm{g}$ of lung after $48 \mathrm{~h}$ of hyperoxia. CoASH levels declined from $6.40 \pm 0.84$ to $3.0 \pm 0.65 \mathrm{nmol} / \mathrm{g}$ of lung, and acetyl CoA levels also were lower in the lungs of animals exposed to hyperoxia. CoASH/CoASSG ratios were lower in animals exposed to hyperoxia, satisfying our previously defined criteria for an oxidant stress on this thiol/disulfide redox couple, but absolute CoASSG levels were not increased, as would be expected for oxidant stresses driven simply by increases in reactive oxygen species or other oxidants. Pulmonary edema was observed in the hyperoxic rats and accounted for some of the declines in CoASH concentrations, but CoASH contents per total lung also declined. Lung mitochondrial succinate dehydrogenase activities were not diminished in rats exposed to hyperoxia, indicating that the decreases in $\mathrm{CoASH}$ concentrations are not attributable to general destruction of lung
\end{abstract}

\section{ABSTRACT}

mitochondria. Lung GSSG contents were greater in the hyperoxia animals, but GSH/GSSG ratios, which are dominated by extramitochondrial pools, did not decrease in these animals. The mechanisms responsible for, and the possible pathophysiologic consequences of, the decreases in lung CoASH concentrations are not evident from the data available at the present time, but the loss of more than half the tissue contents of CoASH is likely to generate additional metabolic effects that could have significant pathophysiologic consequences. (Pediatr Res 51: 346-353, 2002)

CoASH, coenzyme A

\section{Abbreviations}

CoASSG, coenzyme A-glutathione mixed disulfide

CoASSP, coenzyme A-protein mixed disulfides

GSH, glutathione

GSSG, glutathione disulfide

AcCoA, acetyl-CoA

ROOH, lipid hydroperoxides

$\mathbf{H}_{2} \mathbf{O}_{2}$, hydrogen peroxide

PSSG, protein-S-glutathione mixed disulfide

$\mathrm{Fio}_{2}$, fraction of inspired oxygen

SDH, succinate dehydrogenase

PSH, protein thiol

DEPA, diethylenetriaminepentaacetic acid

$\gamma$-glu-glu, $\gamma$-glutamyl-glutamate
Administration of supplemental oxygen remains an important therapy in the care of patients with pulmonary insufficiency, as is encountered in many prematurely born infants or in adults with acute respiratory distress. However, this supplemental oxygen can cause adverse effects, as has been observed

Received August 25, 2000; accepted September 19, 2001

Correspondence and reprint requests: Charles Vincent Smith, Ph.D., Center for Developmental Pharmacology and Toxicology, Children's Research Institute, 700 Children's Drive, Columbus, OH 43205, U.S.A.; e-mail: smithcv@chi.osu.edu in the lungs of experimental animals and humans exposed to elevated oxygen tensions (1-4). Cellular injury is manifested when the rates of generation of the reactive species are increased beyond the capacities of the antioxidant defense mechanisms, such as with exposure to hyperoxia $(1,5,6)$, or when the functions of the antioxidant defense mechanisms are deficient, compromised, or developmentally unprepared, such as in prematurely born infants $(3,7,8)$.

In the course of exposure of experimental animals to $>95 \%$ oxygen, a relatively prolonged period usually is observed in 
which no manifestations of injury or dysfunction are readily apparent. This initiation phase is approximately 24 to $36 \mathrm{~h}$ in adult male Fischer-344 rats and 36-48 h in Sprague-Dawley rats $(2,9)$. After the initiation phase, animals continuously exposed to $>95 \% \mathrm{O}_{2}$ develop rapidly progressing lung dysfunction, which is followed in short order by death. The prolonged initiation phase followed by the rapid onset of the destructive phase is suggestive of a process in which a crucial intracellular pool, presumably of a critical component involving the antioxidant defense mechanisms, has been depleted to a threshold level, below which cell damage and organ dysfunction are observed.

The initiating events of pulmonary oxygen toxicity probably are mediated by reactive oxygen species, such as $\mathrm{H}_{2} \mathrm{O}_{2}$ and $\mathrm{ROOH}$, produced in greater amounts in response to the elevated partial pressures of oxygen (Eq. 1). GSH is distributed throughout mammalian cells and most extracellular fluids and is essential to effective reduction of oxidants, such as $\mathrm{H}_{2} \mathrm{O}_{2}$ and other hydroperoxides (10). Reduction of $\mathrm{H}_{2} \mathrm{O}_{2}$ and other $\mathrm{ROOH}$ by GSH-dependent glutathione peroxidases produces GSSG and water or the respective alcohol and limits alteration of other biologic molecules by the reactive hydroperoxides (Eq. 2). Sustained availability of GSH depends on cellular synthesis of GSH (not shown) and NAPDH-dependent reduction of GSSG by glutathione reductase (Eq. 3).

$$
\begin{aligned}
\mathrm{O}_{2} & \rightarrow \mathrm{ROOH} \\
\mathrm{ROOH}+2 \mathrm{GSH} & \rightarrow \mathrm{ROH}+\mathrm{GSSG} \\
\mathrm{GSSG}+\mathrm{NADPH} & \rightarrow 2 \mathrm{GSH}+\mathrm{NAPD}^{+}
\end{aligned}
$$

Increases in susceptibility to hyperoxic lung injury in experimental animals depleted of GSH or GSH-dependent antioxidant functions have been demonstrated in numerous studies (11-15). However, exposure of experimental animals to hyperoxia has not been found to lead to depletion of GSH (11-16), as would be expected with a GSH threshold-dependent onset of lung injury (17). Although increases in lung GSSG contents concomitant with onset of hyperoxic lung injury have been observed $(18,19)$, the absolute levels of GSSG attained have been limited $(9,16,20,21)$, and significant $S$-thiolation of lung proteins has not been reported (22).

The apparent contradiction between the absence of depletion of lung GSH in response to exposure to hyperoxia and the exacerbation and amelioration of hyperoxic lung injury by manipulations that decrease and increase, respectively, GSH supplies and metabolism suggest compartmentalization or other form of selectivity in the effects of hyperoxia on GSH (23). Lung mitochondria produce reactive oxygen species under normal conditions, and the rates of production increase disproportionately as the $\mathrm{PO}_{2}$ is increased $(6,24)$. Some investigations indicate that the mitochondria are injured relatively early in the course of exposure to hyperoxia $(4,12,25)$. Decreases in GSH contents in lung mitochondria were not observed in experimental animals exposed to hyperoxia (12, 25). In fact, mitochondrial GSH contents were reported to increase during exposure to hyperoxia. Although both studies presented the data as GSH, neither study distinguished GSH from GSSG. The data were obtained using enzyme recycling methods that do not distinguish GSH from GSSG, nor from other forms of mixed disulfides with GSH, except through precipitation of proteins with acid, thereby separating PSSG. In most cases, the vast majority of tissue (GSH + GSSG + GSSP + GSSX) is GSH, but this may not be a good assumption under conditions of oxidative challenge, particularly in mitochondria, because mitochondria do not export excess GSSG as actively as most cells export cytosolic GSSG (26).

Intramitochondrial thiol/disulfide status may be a critical determinant of cellular resistance to adverse effects of oxidant challenges. We have observed protection against oxidant stresses in Chinese hamster ovary cells stably transfected with the cDNA for human glutathione reductase, with a most dramatic protection when the glutathione reductase activities were increased selectively in the mitochondria (27). In other studies, we observed that $\mathrm{H} 441$ cells, which most closely resemble lung Clara cells, were protected from tert-butyl-hydroperoxideinduced cell death (28) or from the cytostatic effects of hyperoxia in vitro (29) by adenovirus-mediated gene transfer of glutathione reductase similarly targeted for expression in the mitochondria. Mitochondrial GSSG, acting through thioldisulfide exchange reactions with PSH in processes reflected by GSH/GSSG ratios, could affect cell viability and function through alterations in protein structure (Eq. 4), such as with the mitochondrial permeability transition pore $(30,31)$.

However, efforts to assess the effects of hyperoxia or other oxidative challenges to mitochondrial thiol/disulfide status in vivo are hampered by the time and manipulations required to isolate mitochondria, relative to the rapid artifactual oxidation, reduction, or metabolism of thiols and disulfides. The best methods to minimize these unwanted reactions use freezeclamping of tissues with liquid nitrogen-cooled aluminum tongs, followed by grinding to a powder under liquid nitrogen, and addition of the powder to acid or thiol alkylating agents such as $\mathrm{N}$-ethylmaleimide. Isolation of mitochondria from freeze-clamped or acid-precipitated tissues is not practical. In contrast with mitochondrial GSH and GSSG, which in most tissues represent only approximately $10 \%$ of the total pool, Siess and coworkers (32) have estimated that $80 \%$ of CoASH in rat liver is intramitochondrial. CoASH and CoASSG undergo thiol/disulfide exchange reactions with GSSG and GSH (Eq. 5) (33), and CoASH and CoASSG can be measured by HPLC in acid supernatants of freeze-clamped tissue, thereby offering a thiol/disulfide biomarker that can be measured with minimum post vivo artifacts and that may reasonably be expected to reflect the intramitochondrial GSH/GSSG redox status (33).

$$
\begin{gathered}
\mathrm{GSSG}+\mathrm{PSH} \rightleftarrows \mathrm{GSH}+\mathrm{PSSG} \\
\mathrm{GSSG}+\mathrm{CoASH} \rightleftarrows \mathrm{GSH}+\mathrm{CoASSG}
\end{gathered}
$$

In the present study, we measured CoASH and CoASSG in freeze-clamped lung tissues from male Fischer-344 and Sprague-Dawley rats exposed to $>95 \% \mathrm{O}_{2}$ for $48 \mathrm{~h}$ and in air-breathing control rats to test the hypothesis that oxidant 
stresses on lung thiol status would cause oxidative shifts in the CoASH/CoASSG redox couple, which, if observed, would be interpreted as preliminary evidence of oxidant stress effects in mitochondria. We compared the effects of hyperoxia in Fischer-344 rats and Sprague-Dawley rats to test the hypothesis that differences in changes in CoASH/CoASSG status would correspond with the greater sensitivity to hyperoxic lung injury of the Fischer-344 rats (9).

\section{METHODS}

Animals. All animal studies were approved by the Baylor College of Medicine Animal Protocol Review Committee. The studies used Sprague-Dawley and Fischer-344 adult male rats weighing 200-265 g obtained from Harlan Industries (Houston, TX, U.S.A.). The rats were housed in a sealed chamber with a 12-h/12-h light-dark cycle, and were permitted free access to food and water throughout the experimental period. The oxygen concentrations in the chamber were monitored twice daily and maintained at $>95 \%$ by the administration of pure oxygen at $5 \mathrm{~L} / \mathrm{min}$. Sodasorb (Dewey \& Almy Chemical Division, Grace \& Co., Lexington, MA, U.S.A.) was placed in the chamber to absorb carbon dioxide. Throughout the study period, both strains were exposed to identical concentrations of oxygen simultaneously in the same sealed chamber, with a partition separating the two strains of rats. Equal numbers of Sprague-Dawley and Fischer-344 rats were killed after $48 \mathrm{~h}$ of exposure to $>95 \%$ oxygen. Also, air-breathing control animals from each strain were killed, and the samples were processed in parallel with samples obtained from the hyperoxia-exposed rats. The rats were anesthetized with $200 \mathrm{mg} / \mathrm{kg}$ of i.p. pentobarbital, a midline thoracotomy was performed, and intracardiac blood samples were obtained. Heparinized syringes were used for the collection of samples used for $\mathrm{Hb}$ determinations. Blood samples for $\mathrm{Hb}$ measurements were frozen at $-70^{\circ} \mathrm{C}$ and analyzed later using Boehringer-Mannheim Diagnostics Cyanmethemoglobin Reagent (Roche Molecular Biochemicals, Indianapolis, IN, U.S.A.). Immediately after the blood samples were collected, portions of lung tissue were freezeclamped with liquid nitrogen-cooled tongs and stored at $-70^{\circ} \mathrm{C}$ until processed for HPLC measurements. Next, tissues were obtained from the left lung, stored at $-70^{\circ} \mathrm{C}$, and used subsequently to measure extravascular lung water contents by wet-to-dry weight determinations as described by Erdmann et al. (34).

Measurements. A piece of the frozen lung tissue was weighed and ground to a powder with a mortar and pestle cooled with liquid nitrogen. To the powder was added $4 \mathrm{~mL}$ of ice-cold $3.0 \% \mathrm{HClO}_{4}$ per gram of tissue, and the mixture was homogenized with a Dounce homogenizer. The homogenate was centrifuged at $10,000 \times g$ for $5 \mathrm{~min}$ at $5^{\circ} \mathrm{C}$. Two hundred microliters of fresh whole blood was added to $40 \mu \mathrm{L}$ of $70 \%$ $\mathrm{HClO}_{4}$, and the resulting mixture was vortexed and centrifuged at $10,000 \times g$ for $5 \mathrm{~min}$ at $5^{\circ} \mathrm{C}$. The $\mathrm{CoA}$ species were analyzed in the resulting supernatants immediately, and aliquots of the supernatants and pellets were stored at $-70^{\circ} \mathrm{C}$ before other analyses.
CoASH, CoASSG, and AcCoA quantitation. Concentrations of CoASH, CoASSG, and AcCoA in blood and lung tissue samples were quantitated by a modification of the HPLC method described King and Reiss (35). A Waters 6000A pump with an Alltech 7- $\mu \mathrm{m} \mathrm{C}_{18}$ Adsorbosphere column was used (Alltech Associates, Deerfield, IL, U.S.A.). Detection was at $254 \mathrm{~nm}$ with a Waters 440 detector. Isocratic conditions using $0.2 \mathrm{M}$ sodium phosphate, $\mathrm{pH} 2.8$, with $4.5 \%$ acetonitrile separated the compounds of interest within $30 \mathrm{~min}$. Standards were prepared in $100 \mathrm{mM}$ sodium phosphate, $\mathrm{pH}$ 3.0. CoASH and AcCoA standards were in the range of 0.5 to $4.0 \mu \mathrm{M}$ and CoASSG standards were in the range of 0.25 to $3.0 \mu \mathrm{M}$. A linear relationship was obtained between the concentrations of $\mathrm{CoASH}, \mathrm{CoASSG}$, and AcCoA and the respective peak areas. The limits of detection were approximately 5 pmol on column for all three standards, at signal-to-noise ratios of 5 (data not shown).

GSH and GSSG quantitation. An HPLC method was used, using fluorimetric detection of GSH and GSSG, after derivatization with dansyl chloride (1-dimethylaminonaphthalene-5sulfonyl chloride) (36). Blood and lung tissue samples were prepared as above, and $100 \mu \mathrm{L}$ aliquots of the acid supernatants were mixed with $100 \mu \mathrm{L}$ of $5 \% \mathrm{HClO}_{4}, 0.2 \mathrm{M}$ boric acid, cresol red indicator (5 mg/L), 2.0 M DEPA buffer (Buffer I), 20 $\mu \mathrm{L}$ of $1.0 \mathrm{mM} \gamma$-glu-glu internal standard, and $25 \mu \mathrm{L}$ of 10 $\mathrm{mM}$ iodoacetic acid. To each solution was added a predetermined amount of $2.0 \mathrm{M} \mathrm{LiOH}$ to give a final $\mathrm{pH}$ of 8.0-8.5. The final $\mathrm{pH}$ was measured in each sample, using colorpHast Indicator Strips (Merck, EM Science, Gibbstown, NJ, U.S.A.). The solutions were placed in the dark for $30 \mathrm{~min}$ at $25^{\circ} \mathrm{C}$. To each sample was added an equal volume of dansyl chloride (1 $\mathrm{mg} / \mathrm{mL}$ in acetonitrile), and the samples were returned to the dark for $60 \mathrm{~min}$. The samples were extracted with equal volumes of chloroform, and the aqueous phases were removed for HPLC analysis. Standards of GSH ( $0-40 \mathrm{nmol}$ per assay) and GSSG (0-4 nmol per assay) were prepared in $50 \mathrm{mM}$ HEPES-2.0 mM DEPA buffer. Mobile phase solvent A contained methanol/water $(4: 1, \mathrm{vol} / \mathrm{vol})$. Solvent B was prepared by adding $150 \mathrm{~mL}$ of a stock solution containing sodium acetate $(272 \mathrm{~g})$, glacial acetic acid $(378 \mathrm{~mL})$, and water $(122$ $\mathrm{mL}$ ) to $850 \mathrm{~mL}$ of solvent A. Separation was accomplished on an Ultracil $\mathrm{NH}_{2}$ column using a flow rate of $1.0 \mathrm{~mL} / \mathrm{min}$ and a linear gradient of $100 \%$ solvent A to $100 \%$ solvent B over 20 min, with a 10 -min hold at $100 \%$ solvent B. Detection was accomplished with an Aminco-Bowman spectrophotofluorimeter detector, using $328 \mathrm{~nm}$ as the excitation wavelength and $541 \mathrm{~nm}$ as the emission wavelength. GSH and GSSG concentrations were estimated by peak area ratios relative to the $\gamma$-glu-glu internal standard, compared with experimentally derived standard curves.

SDH activities. SDH activities were measured spectrophotometrically according to Singer (37). Lung homogenates were fractionated by differential centrifugation, and SDH activities were determined in mitochondrial and cytosolic fractions by monitoring the reduction of ferricyanide at $420 \mathrm{~nm}$.

PSSG determination. PSSG were quantitated using a modification of methods described previously (38). Lung tissue samples were prepared with acid precipitation of homogenates, 
as described above. One milliliter of $100 \%$ ethanol was added to each of the pellets, and the mixtures were sonicated for $30 \mathrm{~s}$. Samples were centrifuged, and the pellets were washed a second time in ethanol. After centrifuging, the protein pellets were suspended in $500 \mu \mathrm{L}$ of $50 \mathrm{mM}$ HEPES-2.0 mM DEPA buffer, $\mathrm{pH} 8.0$, containing $25 \mathrm{mM}$ DTT. The mixtures were sonicated for $30 \mathrm{~s}$ and incubated for $60 \mathrm{~min}$ at $37^{\circ} \mathrm{C}$. At the end of the incubation, $50 \mu \mathrm{L}$ of $70 \% \mathrm{HClO}_{4}$ was added and the solution vortexed for $30 \mathrm{~s}$ and then centrifuged at $10,000 \times g$ for $5 \mathrm{~min}$ at $5^{\circ} \mathrm{C}$. The supernatants were used for the determinations of GSH as described above.

Protein concentrations were determined according to the technique described by Bradford (39).

Statistics. All data are expressed as mean \pm SEM. Data were analyzed using a two-way ANOVA, with strain and $\mathrm{FiO}_{2}$ as the independent variables. The results of these tests are presented as whether the data indicated effects of strain of rat, meaning a statistical tendency for Sprague-Dawley rats to be different from Fischer-344 rats, independent of $\mathrm{FiO}_{2}$; similarly, a notation of an effect of oxygen indicates a variable affected by exposure of the rats to $95 \% \mathrm{O}_{2}$, independent of any effects of the strain of rat. The presence of an interaction indicates a statistically significant difference in the effects of hyperoxia on the two strains of rats, or, viewed differently, differences in the manner in which the two strains of rats respond to differences in $\mathrm{Fio}_{2}(40)$. When the two-way ANOVA detected an effect of strain of rat or of oxygen concentration, expressed as $\mathrm{Fio}_{2}$, or an interaction between these factors, subsequent post hoc testing was performed with modified $t$ tests to determine differences between groups of the same strain, but different $\mathrm{Fio}_{2}$, and within the same oxygen exposures but with different strains. All statistical comparisons were performed using SPSS for Windows (SPSS version 7.0, SPSS, Inc., Chicago, IL, U.S.A.).

\section{RESULTS}

There were no deaths in rats maintained in air or exposed to hyperoxia for up to $48 \mathrm{~h}$. Exposure to hyperoxia led to increases in extravascular lung water contents, and the increases were greater in the Fischer-344 rats than in Sprague-Dawley rats (Fig. 1). There were no interstrain differences in lung GSH or GSSG concentrations (moles per gram wet weight of tissue) in rats maintained in room air (Table 1). Lung GSH concentrations were lower in rats after exposure to hyperoxia for $48 \mathrm{~h}$, and the decreases in lung GSH levels were more pronounced in Fischer-344 rats than in Sprague-Dawley rats. There were no effects of exposure to hyperoxia or of strain on lung GSSG concentrations, or on the lung GSH/GSSG ratios (Table 1). Lung CoASH, CoASSG, and AcCoA concentrations were similar in Fischer-344 and Sprague-Dawley rats and were decreased in the two strains of rats by exposure to hyperoxia (Table 2). The effects of hyperoxia on lung CoASH concentrations were quantitatively greater than were the effects on the CoASSG concentrations, as reflected by significant decreases in the CoASH/CoASSG ratios in animals exposed to hyperoxia (Table 2). The declines in lung concentrations of CoASH are not caused by simple shifts in thiol/disulfide redox equilibria,

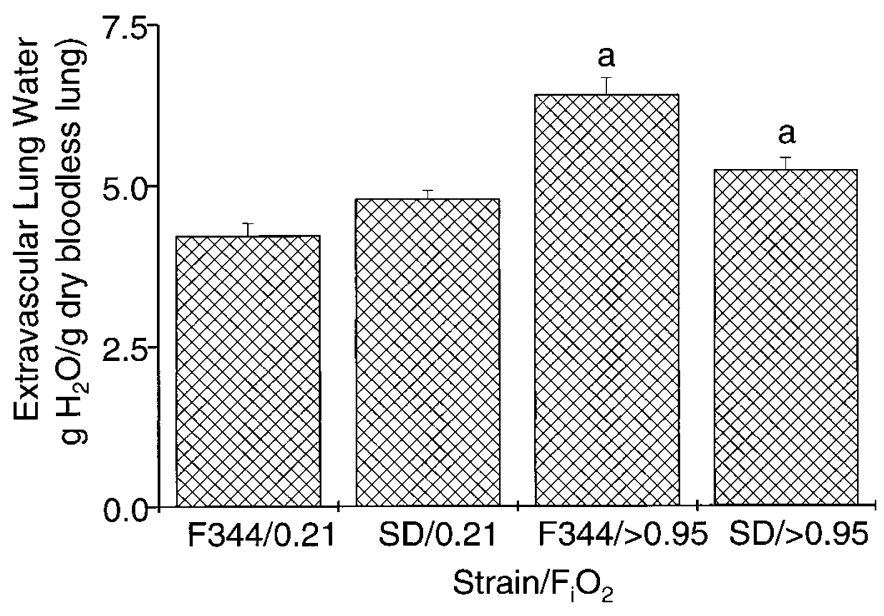

Figure 1. Effects of hyperoxia exposure on extravascular lung water. Fischer344 (F344) and Sprague-Dawley (SD) rats were exposed to $\mathrm{FiO}_{2}$ of 0.21 or $>0.95$ for $48 \mathrm{~h}$, and extravascular lung water contents were measured. Data are expressed as mean \pm SEM, $n=3-5$ per group, and were assessed statistically by two-way ANOVA with differences noted at $p<0.05$. There was a strain-independent effect of $\mathrm{FiO}_{2}$ exposure, but no $\mathrm{O}_{2}$-independent effect of strain. There was a significant interaction between strain and $\mathrm{Fio}_{2}$. Data with common letter superscripts are different from each other.

because the decreases in CoASH concentrations are not accompanied by comparable increases in the levels of CoASSG or AcCoA (Table 2).

To determine the extent to which pulmonary edema (Fig. 1) influenced the decreased lung concentrations of these cofactors in rats exposed to hyperoxia (Tables 1 and 2), the contents of CoA species and of GSH and GSSG were determined per whole lung in a subset of the animals studied. The calculations were not corrected for body weights, but the animal weights in the experimental groups were not different (data not shown). Lung CoASH contents, assessed as nanomoles of CoASH per whole lung, were higher in Sprague-Dawley rats maintained in room air than in air-breathing Fischer-344 rats. Lung CoASH contents were lower in hyperoxia-exposed rats than in rats maintained in room air (Fig. $2 A$ ), but CoASSG contents were not diminished significantly by exposure to hyperoxia, and there were no strain-dependent effects (Fig. 2B). Measurements of SDH activities in subcellular fractions of lung homogenates from animals exposed to hyperoxia and air-breathing control animals did not indicate significant mitochondrial destruction in the animals exposed to hyperoxia (data not shown). These results do not support loss or destruction of mitochondria as an explanation for the decrease in lung concentrations of CoASH.

Lung GSH contents (micromoles per whole lung) were higher in Sprague-Dawley rats than in Fischer-344 rats, but were not altered significantly by exposure to hyperoxia (Fig. $3 A$ ). Lung GSSG contents were increased in lungs of animals exposed to hyperoxia, although there was no effect of strain (Fig. 3B). We measured GSH released from acid-precipitated proteins by reduction with DTT. This entity is frequently termed PSSG, indicating the mixed disulfide of GSH and the cysteine residues on proteins. In rats exposed to hyperoxia, approximately 2 to 3 times as much GSH was observed on reduction from PSSG (Fig. 3C) as was observed in measurements of GSSG (Fig. 3B), which in turn was considerably 
Table 1. Effects of 48 h of hyperoxia on rat lung GSH and GSSG concentrations

\begin{tabular}{lcccc}
\hline Strain & $\mathrm{FiO}_{2}$ & $\begin{array}{c}\mathrm{GSH} \\
(\mu \mathrm{mol} / \mathrm{g} \text { lung })\end{array}$ & $\begin{array}{c}\text { GSSG } \\
(\mu \mathrm{mol} / \mathrm{g} \text { lung })\end{array}$ & GSH/GSSG \\
\hline F344 & 0.21 & $1.56 \pm 0.09$ & $0.072 \pm 0.009$ & $24.5 \pm 4.2$ \\
SD & 0.21 & $1.85 \pm 0.13$ & $0.081 \pm 0.019$ & $33.0 \pm 6.1$ \\
F344 & $>0.95$ & $1.23 \pm 0.11^{\mathrm{a}}$ & $0.093 \pm 0.024$ & $22.5 \pm 6.2$ \\
SD & $>0.95$ & $1.74 \pm 0.13^{\mathrm{a}}$ & $0.092 \pm 0.014$ & $22.0 \pm 4.5$ \\
\hline
\end{tabular}

Fischer-344 (F344) and Sprague-Dawley (SD) rats were exposed to $\mathrm{FiO}_{2}$ of 0.21 or $>0.95$ for $48 \mathrm{~h}$, after which lung GSH and GSSG concentrations were measured per gram of lung tissue. Data are expressed as mean \pm SEM, $n=6-9$ per group, and were assessed statistically by two-way ANOVA with differences noted at $p<0.05$.

* Effect of strain. Data with common letter superscripts are different from each other.

Table 2. Effects of 48 h of hyperoxia on rat lung CoASH, CoASSG, and AcCoA

\begin{tabular}{|c|c|c|c|c|c|}
\hline Strain & $\mathrm{FiO}_{2}$ & $\begin{array}{c}\mathrm{CoASH}^{*} \\
\text { (nmol/g lung) }\end{array}$ & $\begin{array}{c}\text { CoASSG* } \\
\text { (nmol/g lung) }\end{array}$ & $\begin{array}{c}\text { AcCoA* } \\
\text { (nmol/g lung) }\end{array}$ & CoASH/CoASSG* \\
\hline F344 & 0.21 & $6.40 \pm 0.84^{\mathrm{a}}$ & $0.89 \pm 0.15^{\mathrm{c}}$ & $3.14 \pm 0.36^{\mathrm{e}}$ & $9.29 \pm 1.64^{\mathrm{g}}$ \\
\hline F344 & $>0.95$ & $3.00 \pm 0.65^{\mathrm{a}}$ & $0.51 \pm 0.13^{\mathrm{c}}$ & $2.34 \pm 0.43^{\mathrm{e}}$ & $7.08 \pm 1.36^{\mathrm{g}}$ \\
\hline $\mathrm{SD}$ & $>0.95$ & $2.61 \pm 0.44^{\mathrm{b}}$ & $0.61 \pm 0.09^{\mathrm{d}}$ & $1.90 \pm 0.17^{\mathrm{f}}$ & $6.05 \pm 2.33^{\mathrm{h}}$ \\
\hline
\end{tabular}

Fischer-344 (F344) and Sprague-Dawley (SD) rats were exposed to $\mathrm{Fio}_{2}$ of 0.21 or $>0.95$ for $48 \mathrm{~h}$, after which lung concentrations of CoASH, CoASSG, and AcCoA were determined per gram of lung tissue. Data are expressed as mean \pm SEM, $n=9-12$ per group, and were assessed statistically by two-way ANOVA with differences noted at $p<0.05$.

* Effect of $\mathrm{FiO}_{2}$. Data with common letter superscripts are different from each other.

greater than the contents of CoASSG (Fig. 2B). However, exposure to hyperoxia did not alter the lung PSSG contents.

Because of our focus on efforts to minimize possible changes in thiol-disulfide status post vivo, the lungs studied were not perfused to remove blood before freeze-clamping. We measured the whole blood concentrations of GSH and GSSG, to assess possible effects that blood thiol/disulfide contents might contribute to measurements of thiol/disulfide parameters in the lung samples. Group means for whole blood GSH concentrations varied between 1.0 and $1.3 \mathrm{mM}$, and there were no effects of strain or exposure to hyperoxia. Group means for whole blood GSSG concentrations were between 0.07 and 0.11 $\mathrm{mM}$, and there were no effects of strain or exposure to hyperoxia (data not shown). These determinations indicate that variable retention of blood was unlikely to affect our estimates of tissue concentrations of GSH and GSSG.

\section{DISCUSSION}

The idea that hyperoxia would exert an oxidant stress on an organism is hardly revolutionary, but the specific oxidant stress responses that contribute to the initiation of hyperoxic lung injury have not been well characterized. The decreases in $\mathrm{CoASH} / \mathrm{CoASSG}$ ratios satisfy our stated criteria for an oxidant stress on this redox couple $(41,42)$. The absence of comparable changes in GSH/GSSG ratios (Table 1) in the same tissues suggests the possible compartmentation of oxidant stress responses in lung mitochondria. The lack of an increase in total tissue PSSG contents also is consistent with confinement of any thiol/disulfide effects to compartments of limited size. However, we observed increases in lung contents (expressed per whole lung) of GSSG (Fig. $3 B$ ) in animals exposed to hyperoxia, whereas lung contents of CoASSG were not increased in the same animals (Fig. 2B). The absence of increases in lung CoASSG contents or concentrations (per gram of tissue, Table 2) argues against the observed effects on CoASH and CoASSG arising from simple effects from increased production of reactive oxygen species or other oxidants, whether mitochondrially compartmentalized or not.

The presumed effector steps in oxidant responses to increased levels of GSSG or decreased GSH/GSSG ratios are $S$-thiolations of PSH groups. Previous efforts, using derivatization with monobromobimane and separation of the fluorescent thioether proteins by SDS-PAGE, failed to identify specific products of protein $S$-thiolation or other oxidation or alkylation reactions in the lungs of animals exposed to hyperoxia (22). However, in its present form, the experimental approach with monobromobimane-SDS-PAGE will not be robust in detection of the preferential loss of one specific sulfhydryl in a protein containing several thiol groups. The measurements of CoASH and CoASSG, as used in the present studies, therefore, are complementary to our previous broader studies of PSH status using monobromobimane.

The magnitudes of the shifts in CoASH/CoASSG ratios (Table 2) and increases in lung GSSG contents (Fig. 3B) we observed are not large, and the potential biologic significance of these changes or the PSH S-thiolations that the measurements were designed to reflect are unknown. Other investigators have reported lower initial levels and larger fold increases in lung GSSG contents, relative to levels in air-breathing control animals, concomitant with onset of hyperoxic lung injury $(9,16,18-21)$. Differences in the specific animal models and methods of analysis exist in these studies (43).

The decreases in lung CoASH contents cannot be attributed to a simple redox shift to CoASSG, or a metabolic accumulation as AcCoA, because the lung concentrations of these species also decrease in response to exposure to hyperoxia (Table 2). Decreased food intake in the rats exposed to hyperoxia (44) might contribute to the decreases in lung concentra- 
A
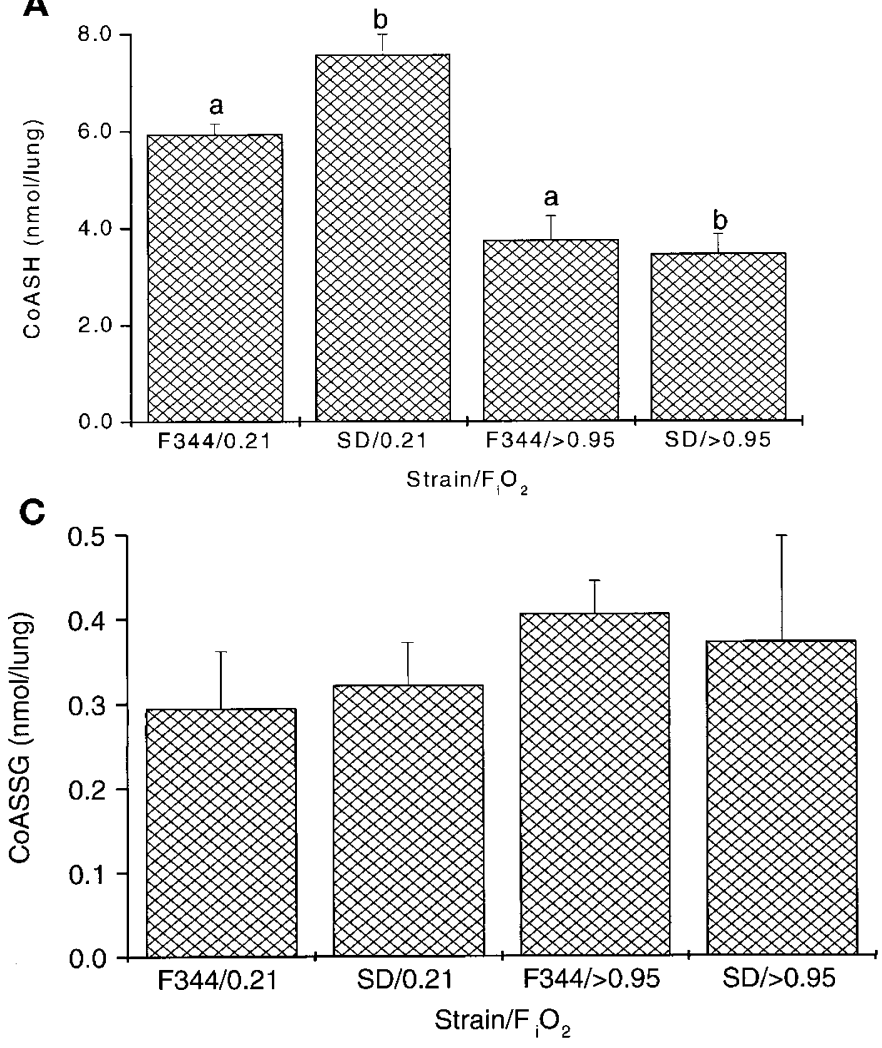

Figure 2. Effects of hyperoxia exposure on total lung contents of CoASH and CoASSG. Fischer-344 (F344) and Sprague-Dawley (SD) rats were exposed to $\mathrm{FiO}_{2}$ of 0.21 or $>0.95$ for $48 \mathrm{~h}$, and contents of CoASH $(A)$ and CoASSG $(B)$ per lung were determined. Data are expressed as mean \pm SEM, $n=3-5$ per group, and were assessed statistically by two-way ANOVA with differences noted at $p<0.05$. Hyperoxia exposure decreased lung contents of $\mathrm{CoASH}$ significantly, whereas total lung CoASSG contents were not decreased, irrespective of strain. Data with common letter superscripts are different from each other.

tions of CoA species. However, fasting alone causes increases in total hepatic CoA species, especially the short-chain acyl CoA esters $(43,45,46)$. Heart, kidney, gastrocnemius, and diaphragm were relatively resistant to changes in CoA contents during a $48 \mathrm{~h}$ fast in rats (45). We are unaware of comparable published data on CoA species in rodent lungs, but in our own experiments, we have observed no significant decline of lung levels of CoASH and CoASSG in rats deprived of food for 24 or $48 \mathrm{~h}$ (data not shown). The marked increases reported in short-chain acyl CoA species observed in livers of rats fasted for 21 to $48 \mathrm{~h} \mathrm{(46)}$ also contrast sharply with the $50 \%$ decreases we observed in levels of $\mathrm{AcCoA}$ in lungs of rats exposed to hyperoxia for $48 \mathrm{~h}$ (Table 2). Diminished food intake alone does not appear to offer a likely explanation for lung CoASH depletion during exposure to hyperoxia, but the possibility of an interaction of diminished food intake and primary effects of hyperoxia deserves additional study.

The pathways and regulation of CoASH degradation are not well-characterized (47). The cellular mechanisms responsible for degradation of CoASH appear to involve initial hydrolysis to the 3'-dephospho derivative by a lysosomal enzyme, which suggests that CoASH turnover depends on lysosomal degradation of whole mitochondria. Lysosomal degradation of whole
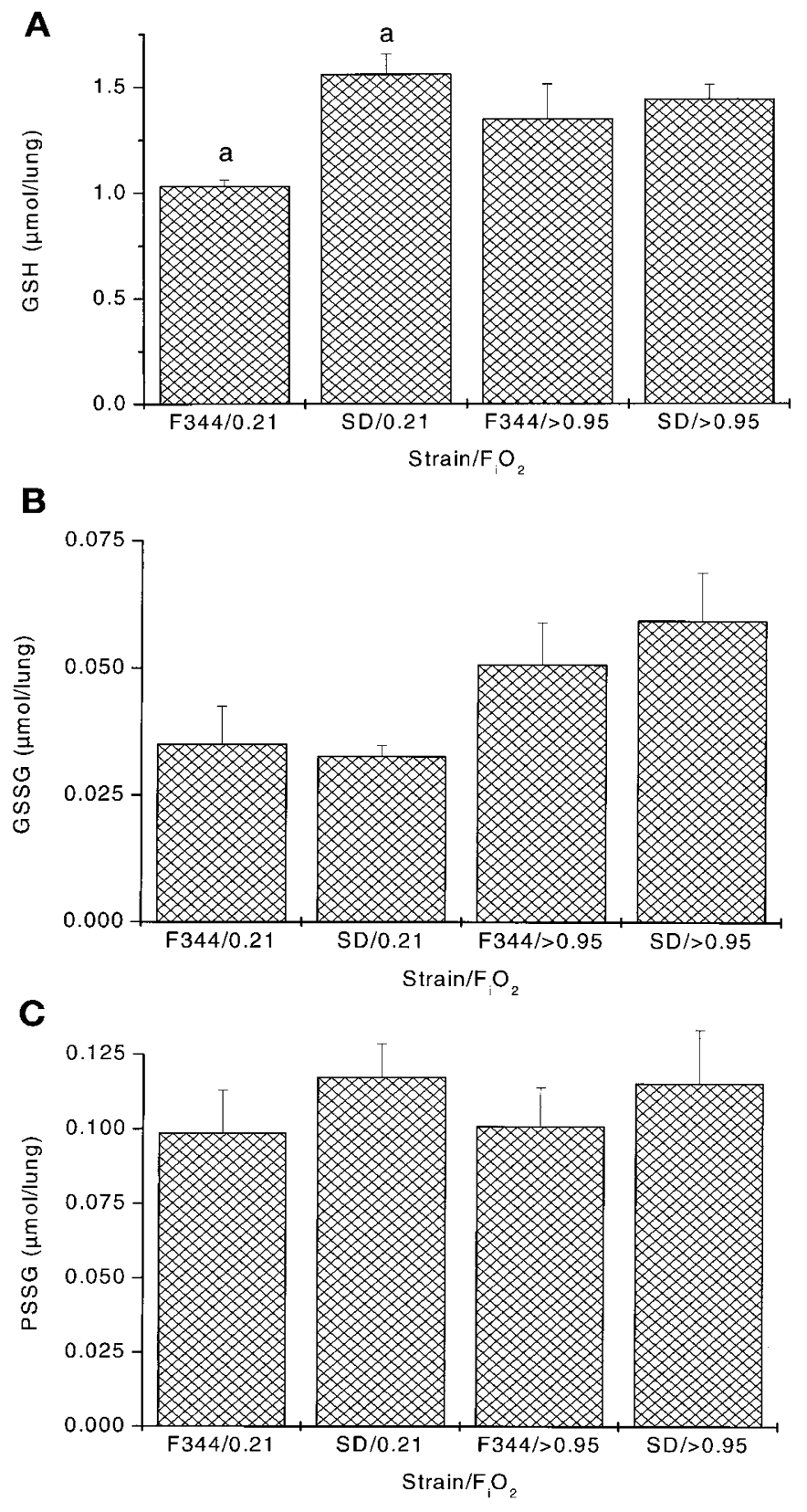

Figure 3. Effects of hyperoxia exposure on lung contents of GSH, GSSG, and PSSG. Fischer-344 (F344) and Sprague-Dawley (SD) rats were exposed to $\mathrm{FiO}_{2}$ of 0.21 or $>0.95$ for $48 \mathrm{~h}$, and GSH $(A)$, GSSG $(B)$, and PSSG $(C)$ contents per lung were determined. Data are expressed as mean $\pm \mathrm{SEM}, n=$ 3-5 per group, and were assessed statistically by two-way ANOVA with differences noted at $p<0.05$. There was no effect of hyperoxia exposure on lung GSH or PSSG contents, whereas hyperoxia exposure increased lung GSSG contents. There was an effect of strain on lung GSH contents. Data with common letter superscripts are different from each other.

mitochondria, such as through autophagocytosis, would not explain the decreases in concentrations of CoASH because of the absence of comparable decreases in SDH activities. Alternatively, extrusion of CoASH from the mitochondria, such as in conjunction with the opening of the mitochondrial permeability transition pore $(30,31)$ or other metabolic changes in production or distribution of CoA species might be responsible for the observed effects. 
The pulmonary edema induced in the animals exposed to hyperoxia will decrease the lung concentrations (expressed per gram of lung or per milligram of protein) of substances not present in comparable concentrations in blood plasma. However, the total lung contents of CoASH also were lower in animals exposed to hyperoxia, meaning that the observed effects on CoASH levels are not explained as a simple dilution in edematous tissues. Decreases in rates of CoASH synthesis also may contribute to the mechanisms responsible for the lower CoASH levels we observed in lungs of hyperoxic animals, but adequate tests of this hypothesis will require turnover studies in the relevant animal models.

Many cell types extrude GSSG and other GS-derived conjugates from the cytosol by means of an ATP-dependent plasma membrane pump (48). Our previous studies in premature infants revealed increased plasma concentrations of GSSG across the heart-lung vascular bed, suggesting net release of GSSG by the lung (8). Mitochondria do not export GSSG as actively as do most cell types studied to date (26), and active export of intramitochondrial CoASSG through this mechanism would not be expected. Depletion of CoASH through formation and cellular extrusion of CoASSG would seem to require formation of the disulfide in the cytosol or a separate mechanism for export of CoASSG from the mitochondria. The potential physiologic consequences of such a process are indicated by the recent report by Schulter et al. (49), who observed that CoASSG caused renal vein constriction in vitro at concentrations as low as $10^{-12} \mathrm{M}$, and increases in mean arterial pressure were observed in intact rats after intraaortic infusion of as little as $50 \mathrm{pmol}$ of CoASSG. The potential of CoASSG thus formed and released to alter pulmonary vascular and bronchial tone in hyperoxia is further suggested by observations of hydroperoxide-induced vaso- and bronchoconstriction in isolated perfused rat lungs (50), but the potency of the effects will necessitate the development of exceedingly sensitive methods for analyses of relevant models in vivo (51).

Protein thiol S-thiolation, with the formation of CoASSP, could contribute to relatively rapid and presumably reversible decreases in levels of soluble CoA species under oxidant stress conditions. Evidence has been reported of the existence of a form of CoA that is tightly bound to mitochondrial proteins and is released by treatment with DTT or $\beta$-mercaptoethanol (52, 53). In their studies, the authors noted significant selectivity in the proteins to which the CoASH was bound, apparently through the reversible formation of disulfide bonds, and they reported partial inhibition of the activities of the CoA-bound forms of the enzymes acetyl-CoA acetyltransferase and 3-oxoacyl-CoA thiolase. The possibility that the reversible protein $S$-thiolation by CoASH serves both as a redox-sensitive enzyme regulatory mechanism and as a mechanism for the mitochondria to retain $\mathrm{CoA}$ is an attractive hypothesis, but will require additional study.

\section{CONCLUSION}

The observed decreases in CoASH/CoASSG ratios in lungs of Fischer-344 and Sprague-Dawley rats exposed to $>95 \% \mathrm{O}_{2}$ for $48 \mathrm{~h}$ satisfy our defined criteria for an oxidant stress.
Because most GSH is extramitochondrial, the absence of a similar oxidant shift of tissue GSH/GSSG ratios suggests a compartmental localization of the forces driving the changes in $\mathrm{CoASH} / \mathrm{CoASSG}$ ratios. However, the absence of increases in absolute levels of CoASSG in the lungs of the rats exposed to hyperoxia is not consistent with the responses anticipated from increased mitochondrial production of reactive oxygen species or other oxidants, and this absence of an increase in absolute concentrations of CoASSG is probably the most important observation in the present studies. The comparable effects of $48 \mathrm{~h}$ of hyperoxia on lung CoA contents in Fischer-344 and Sprague-Dawley rats do not correlate with the greater lung injury in the Fischer-344 rats, suggesting that other factors are the dominant determinants of the differences in strain sensitivities to hyperoxic lung injury. Additionally, the link between $\mathrm{CoASH} / \mathrm{CoASSG}$ ratios and intramitochondrial redox status is speculative. The fractional distribution of CoA species in intraand extramitochondrial spaces and any differences in distribution based on cell type, nutritional status, and metabolic challenges imposed by hyperoxia or other toxicants have not been characterized. Nevertheless, the decreases in lung CoASH concentrations in the rats exposed to hyperoxia should prove to be interesting mechanistically, and may prove to be significant pathophysiologically, but these questions will require further investigation.

\section{REFERENCES}

1. Freeman BA, Tanswell AK 1985 Biochemical and cellular aspects of pulmonary oxygen toxicity. Adv Free Rad Biol Med 1:133-164

2. Crapo JD 1986 Morphologic changes in pulmonary oxygen toxicity. Ann Rev Physiol 48:721-731

3. Frank L 1991 Developmental aspects of experimental pulmonary oxygen toxicity. Free Rad Biol Med 11:463-494

4. Crapo JD, Barry BE, Foscue HA, Shelburne J 1980 Structural and biochemical changes in rat lungs occurring during exposures to lethal and adaptive doses of oxygen. Am Rev Respir Dis 122:123-143

5. Wispe JR, Roberts RJ 1987 Molecular basis of pulmonary oxygen toxicity. Clin Perinatol 14:651-666

6. Turrens JF, Freeman BA, Crapo JD 1982 Hyperoxia increases $\mathrm{H}_{2} \mathrm{O}_{2}$ release by lung mitochondria and microsomes. Arch Biochem Biophys 217:411-421

7. deLemos RA, Coalson JJ 1992 The contributions of experimental models to our understanding of the pathogenesis and treatment of bronchopulmonary dysplasia. Clin Perinatol 19:521-539

8. Smith CV, Hansen TN, Martin NE, McMicken HW, Elliott SJ 1993 Oxidant stress responses in premature infants during exposure to hyperoxia. Pediatr Res 34:360-365

9. Stenzel JD, Welty SE, Benzick AE, Smith EO, Smith CV, Hansen TN 1993 Hyperoxic lung injury in Fischer-344 and Sprague-Dawley rats in vivo. Free Radic Biol Med 14:531-539

10. Meister A 1991 Glutathione deficiency produced by inhibition of its synthesis, and its reversal: applications in research and therapy. Pharm Ther 51:155-194

11. Langley SC, Kelly FJ 1992 Effect of food restriction on hyperoxia-induced lung injury in preterm guinea pig. Am J Physiol 263:L357-L362

12. Smith LJ, Anderson J 1992 Oxygen-induced lung damage: relationship to lung mitochondrial glutathione levels. Am Rev Respir Dis 146:1452-1457

13. Deneke SM, Gershoff SN, Fanburg BL 1983 Potentiation of oxygen toxicity in rats by dietary protein or amino acid deficiency. J Appl Physiol 54:147-151

14. Deneke SM, Lynch BA, Fanburg BL 1985 Transient depletion of lung glutathione by diethylmaleate enhances oxygen toxicity. J Appl Physiol 58:571-574

15. Deneke SM, Lynch BA, Fanburg BL 1985 Effects of low protein diets or feed restriction on rat lung glutathione and oxygen toxicity. J Nutr 115:726-732

16. Jenkinson SG, Black RD, Lawrence RA 1988 Glutathione concentrations in rat lung bronchoalveolar lavage fluid: effects of hyperoxia. J Lab Clin Med 112:345-351

17. Smith CV, Mitchell JR 1989 Pharmacological aspects of glutathione in drug metabolism. In: Dolphin D, Poulson R, Avramovic O (eds) Coenzymes and Cofactors. John Wiley and Sons, New York, pp 1-44

18. White CW, Mimmack RF, Repine JE 1986 Accumulation of lung tissue oxidized glutathione (GSSG) as a marker of oxidant induced lung injury. Chest 89:1115-1135

19. Beehler CJ, Simchuk ML, Toth KM, Drake SK, Parker NB, White CW, Berger EM, Sanderson RJ, Repine JE 1989 Blood sulfhydryl level increases during hyperoxia: a marker of oxidant lung injury. J Appl Physiol 67:1070-1075

20. Groneck P, Gotze-Speer B, Oppermann M, Eiffert H, Speer CP 1994 Association of pulmonary inflammation and increased microvascular permeability during the devel- 
opment of bronchopulmonary dysplasia: a sequential analysis of inflammatory mediators in respiratory fluids of high-risk preterm neonates. Pediatrics 93:712-718

21. Ramsay PL, Wafelman LS, Rogers LK, Smith CV, Welty SE 1996 The effects of dexamethasone (DEX) and hyperoxia on the glutathione (GSH) system in rats. Pediatr Res 39:347A(abstr)

22. Awasthi S, Gyurasics A, Knight SA, Welty SE, Smith CV 1998 Protein oxidation biomarkers in hyperoxic lung injury in rats: effects of U-74389. Toxicol Lett 95:47-61

23. Smith CV, Jones DP, Guenthner TM, Lash LH, Lauterburg BH 1996 Compartmentation of glutathione: implications for the study of toxicity and disease. Toxicol Appl Pharmacol 140:1-12

24. Freeman BA, Crapo JD 1981 Hyperoxia increases oxygen radical production in rat lungs and lung mitochondria. J Biol Chem 256:10986-10992

25. Bassett DJP, Reichenbaugh SS 1992 Lung mitochondrial function following oxygen exposure and diethyl maleate-induced depletion of glutathione. Toxicol Appl Pharmacol 115:161-167

26. Olafsdottir K, Reed DJ 1988 Retention of oxidized glutathione by isolated rat liver mitochondria during hydroperoxide treatment. Biochim Biophys Acta 964:377-382

27. Tamura T, McMicken HW, Smith CV, Hansen TN 1996 Overexpression of human glutathione reductase in Chinese hamster ovary cells protects cells from oxidant injury. Pediatr Res 39:248A(abstr)

28. O’Donovan DJ, Katkin JP, Tamura T, Husser R, Xu X, Smith CV, Welty SE 1999 Gene transfer of mitochondrially targeted glutathione reductase protects $\mathrm{H} 441$ cells from $t$-butyl hydroperoxide-induced oxidant stresses. Am J Respir Cell Mol Biol 20:256-263

29. O'Donovan DJ, Katkin JP, Tamura T, Smith CV, Welty SE 2000 Attenuation of hyperoxia-induced growth inhibition in $\mathrm{H} 441$ cells by gene transfer of mitochondrially targeted glutathione reductase. Am J Respir Cell Mol Biol 22:732-738

30. Petronilli V, Costantini P, Scorrano L, Colonna R, Passamonti S, Bernardi P 1994 The voltage sensor of the mitochondrial permeability transition pore is tuned by the oxidation-reduction state of vicinal thiols. J Biol Chem 269:16638-16642

31. Reed DJ, Savage MK 1995 Influence of metabolic inhibitors on mitochondrial permeability transition and glutathione status. Biochim Biophys Acta 1271:43-50

32. Siess EA, Brocks DG, Wieland OH 1978 Distribution of metabolites between the cytosolic and mitochondrial compartments of hepatocytes isolated from fed rats. Hoppe-Seyler's Z Physiol Chem 359:785-798

33. Gilbert HF 1995 Thiol/disulfide exchange equilibria and disulfide bond stability. Meth Enzymol 251:8-28

34. Erdman AJ, Vaughan TRI, Brigham KL, Woolverton WC, Staub NC 1975 Effect of increased vascular pressure on lung fluid balance in unanesthetized sheep. Circ Res 37:271-284

35. King MT, Reiss PD 1985 Separation and measurement of short-chain coenzyme-A compounds in rat liver by reversed-phase high-performance liquid chromatography. Anal Biochem 146:173-179
36. Martin J, White IN 1991 Fluorimetric determination of oxidised and reduced glutathione in cells and tissues by high-performance liquid chromatography following derivatization with dansyl chloride. J Chromatogr 568:219-225

37. Singer TP 1974 Succinate dehydrogenase. In: Glick D (ed) Methods of Biochemical Analysis. John Wiley and Sons, New York, pp 123-175

38. Livesay JC, Reed DJ 1984 Measurement of glutathione-protein mixed disulfides. Int J Radiation Oncology Biol Phys 10:1507-1510

39. Bradford MM 1976 A rapid and sensitive method for the quantitation of microgram quantities of protein utilizing the principle of protein-dye binding. Anal Biochem 72:248-254

40. Zar JH 1984 Biostatistical Analysis. Prentice-Hall, Englewood Cliffs, pp 206-214

41. Smith CV 1991 Correlations and apparent contradictions in assessment of oxidant stress status in vivo. Free Radic Biol Med 10:217-224

42. Kehrer JP, Smith CV 1994 Free radicals in biology: sources, reactivity and role in the etiology of human diseases. In: Frei B (ed) Natural Antioxidants in Human Health and Disease. Academic Press, New York, pp 25-62

43. Rogers LK, Valentine CJ, Szczypka M, Smith CV 2000 Effects of hepatotoxic doses of acetaminophen and furosemide on tissue concentrations of CoASH and CoASSG in vivo. Chem Res Toxicol 13:873-882

44. Rusakow LS, White CW, Stabler SP $1993 \mathrm{O}_{2}$-induced changes in lung and storage pool thiols in mice: effect of superoxide dismutase. J Appl Physiol 74:989-997

45. Reibel DK, Wyse BW, Berkich DA, Palko WM, Neely JR 1981 Effects of diabetes and fasting on pantothenic acid metabolism in rats. Am J Physiol 240:E597-E601

46. Smith CM, Cano ML, Potyraj J 1978 The relationship between metabolic state and total CoA content of rat liver and heart. J Nutr 108:854-862

47. Robishaw JD, Neely JR 1985 Coenzyme A metabolism. Am J Physiol 248:E1-E9

48. Ishikawa T 1992 The ATP-dependent glutathione $S$-conjugate export pump. Trends Biochem Sci 17:463-468

49. Schulter H, Meissner M, van der Geit M, Tepel M, Bachmann J, Grob I, Nordhoff E, Karas M, Witzel H, Zidek W 1995 Coenzyme A glutathione disulfide: a potent vasoconstrictor derived from the adrenal gland. Circ Res 76:675-680

50. Olafsdottir K, Atzori L, Ryrfeldt A, Berggren M, Kumlin M, Moldeus P 1991 Mechanisms of hydroperoxide-induced broncho- and vasoconstriction in isolated and perfused rat lung. Pharm Tox 68:181-186

51. Atzori L, Olafsdottir K, Corriga AM, Bannenberg G, Ryrfeldt A, Moldeus P 1991 Thiol modification in $\mathrm{H}_{2} \mathrm{O}_{2}$ - and thromboxane-induced vaso- and bronchoconstriction in rat perfused lung. J Appl Physiol 71:1309-1314

52. Huth W, Arvand M, Moller U 1988 Identification of $\left[{ }^{1-14} \mathrm{C}\right]$ pantothenic-acidmediated modified mitochondrial proteins. Eur J Biochem 172:607-614

53. Huth W, Worm-Breitgoff C, Moller U, Wunderlich I 1991 Evidence for an in vivo modification of mitochondrial proteins by coenzyme A. Biochim Biophys Acta 1077:1-10 